\title{
Supporting information for: Liquid Phase Electron Microscopy Provides Opportunities in Polymer Synthesis and Manufacturing
}

\author{
Wyeth Gibson ${ }^{1}$ and Joseph P. Patterson ${ }^{12 *}$ \\ ${ }^{1}$ Department of Chemistry, University of California, Irvine, Irvine, CA 92697-2025 \\ ${ }^{2}$ Department of Materials Science and Engineering, University of California, Irvine, Irvine, $C A$ \\ 92697-2025
}

Figure 4 compares the electron incident energy and approximate electron fluxes for common Ebeam and EM-beam systems. This information for this figure was obtained from industry sources and calculated by making conservative approximations concerning the area the E-beam covers in one second. Calculations use the manufacturer advertised currents. These currents are generally reported at or close to the electron source. Therefore, this does not take into account any inefficiencies between the electron source and the sample which can vary from E-beam models.

Fluences are the only sample independent way to compare beam sources. This comparison still not perfect as the fluence in EM-beams is homogenous and E-beam systems is nonhomogeneous. Fluxes and normalized in terms of area. E-beams tend to have a much lower fluence than the typical EM-beam. However, it is important to remember that absorbed dose is a function of sample thickness meaning that if the sample is thicker the absorbed dose per unit of time in the lower fluence E-beams system may be higher than that of the high fluence TEM with a thin sample.

When looking at industrial E-beam sources there is no standard way in which the fluence or flux is reported. In general, one of three things is given: power in watts, Amps, and processing rates. Processing rates are reported in $\mathrm{kGy} \mathrm{m} \mathrm{s}^{-1} .^{1}$

$$
\text { Area processing rate }=W_{b} V_{1}=K I / D
$$

where $W_{b}=$ beam width in $m ; V_{I}=$ line speed in $\mathrm{m} / \mathrm{min} ; \mathrm{I}=$ beam current in $\mathrm{mA} ; \mathrm{D}=$ dose in $\mathrm{kGy}$. $\mathrm{K}$ is the processing coefficient. $\mathrm{K}$ is source dependent and takes into account the specific irradiation geometries and the energy deposited per electron. $K$ is found empirically for the specific E-beam source. Comparison of E-beam with EM-beam is dependent on the particular E-beam source and the processing coefficient.

To relate advertised E-beam source parameters we calculated the approximated electron flux. This is because the absorbed dose is sample dependent. The following assumptions were made to calculate these fluxes:

1) Dose is calculated at the sample surface, where the surface would be $1 \mu \mathrm{m}$ of water

2) If max dose is given it is at the slowest web speed reported

3) The area irradiated is the reported at the max beam width, $x 1$ meter. 
The choice to use 1 meter is conservative as many of these sources irradiate areas smaller than this but it may be the case that some are larger such as the Rhodotron ${ }^{\oplus}$. Therefore, E-beam sources likely have higher fluxes than those given in Table S1 and Figure 4. If processing rate and power/Amps were given, then processing rate was chosen for the flux calculations.

Table S1. Electron Flux of E-beam and EM-beam sources

\begin{tabular}{|c|c|c|c|c|}
\hline Name & $\begin{array}{l}\text { Electron } \\
\text { Energies }\end{array}$ & $\begin{array}{c}\text { Flux } \\
\left(\mathrm{e}^{-} \mathrm{m}-{ }^{2} \mathrm{~s}^{-1}\right)\end{array}$ & Dose rate & Amps/kW \\
\hline $\begin{array}{c}\text { SEM }^{2} \\
\text { Areas } \\
\text { used: } \\
1 \mathrm{~mm}^{2} \\
0.8 \mu^{2}\end{array}$ & $0.2-40 \mathrm{keV}$ & $3.7 \times 10^{12}-1.7 \times 10^{23}$ & & $0.8 p A-100 n A$ \\
\hline $\begin{array}{c}\text { S/TEM } \\
\left(\mathrm{e}^{-} \mathrm{A}^{-2} \mathrm{~s}^{-1}\right) \\
0.01- \\
1 \mathrm{X} 10^{6}\end{array}$ & $\begin{array}{c}80 \mathrm{keV}-1 \mathrm{MeV} \\
\text { Common: } \\
\text { (200 or } 300 \mathrm{keV} \\
\text { TEM } \\
80 \mathrm{keV} \text { STEM) }\end{array}$ & $2.5 \times 10^{18}-1 \times 10^{26}$ & & \\
\hline E-beam ${ }^{3}$ & $80 \mathrm{kV}$ & $4.92 \times 10^{17}$ & & $\begin{array}{c}2.7 \mathrm{~kW} \\
28.4 \mathrm{~mA}\end{array}$ \\
\hline E-beam ${ }^{4}$ & $80-100 \mathrm{kV}$ & $4.3 \times 10^{15}-5.4 \times 10^{15}$ & 30 kGy @ 183 m/min & \\
\hline E-beam $^{5}$ & $80-300 \mathrm{kV}$ & $\begin{array}{l}1.8 \times 10^{16}- \\
6.8 \times 10^{17}\end{array}$ & $\begin{array}{l}130 \text { kGy @ } 400 \\
m / m i n\end{array}$ & $\begin{array}{l}150-600 \mathrm{~kW} \\
500 \mathrm{~mA}-2 \mathrm{~A}\end{array}$ \\
\hline E-beam ${ }^{6}$ & $2-10 \mathrm{MeV}$ & $1.2 \mathrm{E}^{16}-5 \mathrm{e}^{17}$ & & $\begin{array}{c}20-560 \mathrm{~kW} \\
2-80 \mathrm{~mA}\end{array}$ \\
\hline E-beam ${ }^{7}$ & $100-300 \mathrm{keV}$ & $\begin{array}{l}3.1 \times 10^{17}- \\
9.4 \times 10^{17}\end{array}$ & 300 kGy @ 3 m/min & \\
\hline E-beam ${ }^{1}$ & $0.5-5 \mathrm{MeV}$ & $3 \times 10^{17}-3 \times 10^{18}$ & & $\begin{array}{c}250 \mathrm{~kW} \\
50-500 \mathrm{~mA}\end{array}$ \\
\hline \multicolumn{5}{|c|}{$\begin{array}{l}\text { All fluxes have been calculated and are approximations. All other information was gathered } \\
\text { from advertised E-beam literature. }\end{array}$} \\
\hline & ${ }^{*} \mathrm{~S} / \mathrm{TEM}$ ele & ons flux have been & athered from own data & \\
\hline
\end{tabular}




\section{Example calculations:}

Example calculation from reported Amps:

\begin{tabular}{|l|l|}
\hline Values for the ebeam Compact $^{3}$ & $2.27 \mathrm{~kW}$ \\
\hline Max beam power & $360 \mathrm{~mm}$ \\
\hline Max beam width &
\end{tabular}

Convert watts to amps:

$\mathrm{I}=\mathrm{P} \mathrm{V}^{-1}$

$$
28.4 \times 10^{-3} \mathrm{~A}
$$

Convert to flux:

Assuming area $=1 \mathrm{~m} \times 0.36 \mathrm{~m}=0.36 \mathrm{~m}^{2}$

Amps to electrons $\mathrm{s}^{-1}: 1 \mathrm{Amp}=6.242 \times 10^{18}$ electrons s$^{-1}$

$$
28.4 \times 10^{-3} \mathrm{~A}=\underline{4.92 \times 10^{17} \text { electrons } \mathrm{s}^{-1} \mathrm{~m}^{-2}}
$$

Example calculation from values given in $\mathrm{kGy} \mathrm{m} \mathrm{s}^{-1}$

\begin{tabular}{|c|c|}
\hline \multicolumn{2}{|c|}{ Values for the PCT pilot line $^{7}$} \\
\hline Reported keV & $100-300$ \\
\hline Max web width & $600 \mathrm{~mm}$ \\
\hline Line speed & $3-180 \mathrm{~m} / \mathrm{min}$ \\
\hline Max single pass dose & $300 \mathrm{kGy}$ \\
\hline
\end{tabular}

Assumptions made: Max dose is calculated at max web width and the slowest line speed of 3 $\mathrm{m} / \mathrm{min}$. Also, that dose is calculated at the surface of water which is 1 um thick.

Convert to kGy $\mathrm{m} \mathrm{s}^{-1}$ :

$$
300 \mathrm{kGy} * 3 \mathrm{~m} \mathrm{~min}^{-1}=15 \mathrm{kGy} \mathrm{m} \mathrm{s}^{-1}
$$

Convert to $\mathrm{J} \mathrm{s}^{-1}$ :

$\mathrm{Gy}=\mathrm{J} \mathrm{kg}$

$$
15 \mathrm{kGy} \mathrm{m} \mathrm{s}^{-1}=15 \mathrm{~kJ} \mathrm{~m} \mathrm{~kg}^{-1} \mathrm{~s}^{-1}
$$

Assumed web area irradiated $=1 \mathrm{~m} \times 0.6 \mathrm{~m}$ and depth is $1 \times 10^{-6} \mathrm{~m}$ and using $1000 \mathrm{~kg} \mathrm{~m}^{-3}$ conversion for a total of $6 \times 10^{-4} \mathrm{~kg}$

$$
15 \mathrm{~kJ} \mathrm{~m} \mathrm{~kg}^{-1} \mathrm{~s}^{-1}=9 \mathrm{~J} \mathrm{~s}^{-1}
$$


Convert to Amps

$\mathrm{J} / \mathrm{s}=\mathrm{V}^{*} \mathrm{~A} \quad$ using both $100 \mathrm{kV}$ and $300 \mathrm{kV}$

$$
9 \mathrm{~J} \mathrm{~s}^{-1}=9.0 \times 10^{-5} \mathrm{~A}(100 \mathrm{kV}) \text { and } 3.0 \times 10^{-5} \mathrm{~A}(300 \mathrm{kV})
$$

Convert Amps to number of electrons:

$1 \mathrm{Amp}=6.242 \times 10^{18}$ electrons $\mathrm{s}^{-1}$

$$
\begin{aligned}
& 3.0 \times 10^{-5} A=1.87 \times 10^{14} \text { electrons } \mathrm{s}^{-1} \\
& 9.0 \times 10^{-5} A=5.62 \times 10^{14} \text { electrons } \mathrm{s}^{-1}
\end{aligned}
$$

Calculate electron flux (electrons $\mathrm{m}^{-2} \mathrm{~s}^{-1}$ ):

Assumed area irradiated $=0.6 \mathrm{~m}^{2}$

$$
\begin{aligned}
& 1.87 \times 10^{14} \text { electrons } \mathrm{s}^{-1}=\underline{3.12 \times 10^{14} \text { electrons } \mathrm{m}^{-2} \mathrm{~s}^{-1}} \\
& 5.62 \times 10^{14} \text { electrons } \mathrm{s}^{-1}=\underline{9.36 \times 10^{14} \text { electrons } \mathrm{m}^{-2} \mathrm{~s}^{-1}}
\end{aligned}
$$

\begin{tabular}{|c|c|c|c|}
\hline Source & $\begin{array}{l}\text { Electron } \\
\text { energy }\end{array}$ & $\begin{array}{l}\text { Calculated } \\
\text { flux }\end{array}$ & Use \\
\hline SEM $^{2}$ & $\begin{array}{l}0.2-40 \\
\mathrm{keV}\end{array}$ & $\begin{array}{l}3.7 \times 10^{12}- \\
1.7 \times 10^{23}\end{array}$ & $\begin{array}{l}\text { secondary and backscattered electrons for high } \\
\text { resolution surface analysis }\end{array}$ \\
\hline S/TEM* & $\begin{array}{l}80 \mathrm{keV}- \\
1 \mathrm{MeV}\end{array}$ & $\begin{array}{l}2.5 \times 10^{18} \\
1 \times 10^{26}\end{array}$ & $\begin{array}{l}\text { Transmitted electrons for high resolution projection } \\
\text { images }\end{array}$ \\
\hline E-beam ** & $\begin{array}{l}\text { 80-300 } \\
\text { keV }\end{array}$ & $\begin{array}{l}5.4 \times 10^{15} \\
9.4 \times 10^{17}\end{array}$ & Surface Curing \\
\hline E-beam** & $\begin{array}{l}0.3-3 \\
\mathrm{MeV}\end{array}$ & $\begin{array}{l}9 \times 10^{16} \\
3 \times 10^{18}\end{array}$ & $\begin{array}{c}\text { Shrink Film } \\
\text { Wire and Cable }\end{array}$ \\
\hline E-beam ${ }^{\star *}$ & $\begin{array}{l}3-10 \\
\mathrm{MeV}\end{array}$ & $\begin{array}{l}1 \times 10^{17}- \\
3 \times 10^{18}\end{array}$ & Sterilization \\
\hline \multicolumn{4}{|c|}{$\begin{array}{l}\text { Electron flux and energies were assembled from Table S1. } \\
\text { EM electrons flux have been gathered from the authors own data } \\
\text { energy, typical penetrations, and end uses taken from Cleland et. al.. }\end{array}$} \\
\hline
\end{tabular}

If the pilot line was to be included by itself in Figure 4 the calculated fluxes and reported voltages would be the boundaries of each box. It must be noted that the flux with change at different acceleration voltages.

Table S2. E-beam and EM-beam categories and associated boundaries used for Figure 4

\section{Citations}


1. Cleland, M. R. B., Antony J.; Auslender, V.L.; Bol, J.L.; Brinston, R.; Bryazgin, A.; Calvo, W.A.P.; Chmielewski, A.; Cokragan, A; Ehlermann, D.A.E.; Fletcher, M.; Guven, O.; Haji-Saeid, M.; Hamm, R. W.; Letournel, E.; Machi, S.; Malcom, F.; Mastro, N.L.del,; Safrany, A.; Sampa, M.H.O.; Tallentire, A.; Testoni, A.; Vroom, D.A; Whitney, W.; Zimek, Z. Industrial Radiation with Electron Beams and X-rays; International Irradiation Association: Shropshire, UK, 2011.

2. Verios 5 XHR SEM Datasheet. https://assets.thermofisher.com/TFS-

Assets/MSD/Datasheets/verios5-datasheet.pdf (accessed 20 Feb 2021).

3. ebeam Compact Brochure. https://www.radsys.eu/wp-

content/uploads/2018/10/170614 ebeam-Compact-Flyer.pdf (accessed Feb 23 2021).

4 . Ebeam Core 100 Brochure. https://www.pctebi.com/wp-content/uploads/2019/06/Core100-Handout.pdf (accessed 20 Feb 2021).

5. Invictus Series Ebeam Systems Brochure. https://www.pctebi.com/wpcontent/uploads/2019/06/Invictus-Series-Handout.pdf (accessed 20 Feb 2021).

6. Second Generation Rhodotron® E-beam Accelerator. https://www.ibaindustrial.com/accelerators\#rhodotron-e-beam-accelerator (accessed 20 Feb 2021). 7. Ebeam Pilot Line Brochure. https://www.pctebi.com/ebeam-systems/pilot-line/ (accessed 20 Feb 2021). 